\title{
The Synthesis and Characterization of a Novel Cationic Asphalt Emulsifier
}

\author{
Xue-Di Gong, Lai-Shun Shi ${ }^{\text {a }}$, Tian-Yao Wang, Xiao-Meng Yu \\ School of Chemistry and Chemical Engineering, Shandong University, Jinan 250061, P. R. China
}

\begin{abstract}
A new cationic asphalt emulsifier of N,N-dimethyl-N-(3-(N',N'-dimethyl amido)-2-hydroxy propyl)coconut oil amide propyl-1-ammonium chloride was synthesized by two steps reaction of coconut oil acyl propyl dimethyl tertiary amine (PKO), epoxy chloropropane and dimethylamine. The chemical structure of the key intermediate of N,N-dimethyl-N-(ethylene oxide-2-methylene)-coconut oil amide propyl-1-ammonium chloride was confirmed by FTIR, ${ }^{1} \mathrm{H}$ NMR and elemental analysis. The optimum reaction condition of first step was obtained by single factor analysis: reaction time $5 \mathrm{~h}$, reaction temperature $50{ }^{\circ} \mathrm{C}$, feedstock mole ratio of epoxy chloropropane to PKO 1.05. The reaction yield is $82.15 \%$ and the epoxy value is $40.39 \%$ at the optimum conditions. The critical micelle concentration (CMC) of the asphalt emulsifier is $7.80 \times 10^{-2} \mathrm{~mol} / \mathrm{L}$. The surface tension at CMC is 24.57 $\mathrm{mN} / \mathrm{m}$. The emulsifier showed excellent emulsification effect for the asphalt. The prepared bituminous emulsion had higher storage stability. The emulsifier belongs to medium-set asphalt emulsifier.
\end{abstract}

\section{Introduction}

Emulsified asphalt is composed of asphalt, emulsifier, water and other additives. Compared with the hot asphalt, emulsified asphalt can be applied at room temperature, has the advantages of better adhesive ability with stone, low energy consumption and less environmental pollution. It is widely used in highway and waterproof materials $[1,2]$.

Emulsified asphalt is an oil-in-water type emulsion. Asphalt emulsifier can be classified into cationic, anionic, amphoteric and non-ionic $[3,4]$. At the beginning, the mainly used asphalt emulsifier was anionic, such as long chain sulfonate, the alkali metal salt of lignin, and carboxylic acid salt, etc [5]. Cationic emulsifier has developed rapidly in recent years [6,7]. Cationic asphalt emulsifier has good emulsification performance and strong adhesion force with stone. Most of the stone has a negative charge. Cationic emulsified asphalt can rapid adsorb and bind on the surface of stone. The mainly cationic emulsifiers are nitrogen containing organic amine derivatives and quaternary ammonium salt. Li et al [8] synthesized an asymmetric Gemini cationic surfactants, characterized its structure and discussed the affect factors of stability. Banno et al [9] synthesized novel cationic surfactants which have biodegradability and chemical recyclability green sustainable.

In previous papers, different kinds of asphalt emulsifiers were synthesized by our research group [7,1014]. A new cationic asphalt emulsifier of N,N-dimethyl$\mathrm{N}-\left(3-\left(\mathrm{N}^{\prime}, \mathrm{N}^{\prime}\right.\right.$-dimethyl amido)-2-hydroxy propyl)-coconut oil amide propyl-1-ammonium chloride was synthesized by two steps reaction of coconut oil acyl propyl dimethyl

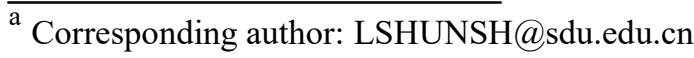

tertiary amine (PKO), epoxy chloropropane and dimethylamine in this paper. The structure of the emulsifier was confirmed by FTIR, ${ }^{1} \mathrm{H}$ NMR and elemental analysis. The emulsifier belongs to medium-set asphalt emulsifier.

\section{Experimental}

\subsection{Materials and characterization}

Coconut oil acyl propyl dimethyl tertiary amine (PKO) was technical grade and obtained from Lianyungang Yihai Chemical Co., Ltd, Lianyungang, China. The other chemical reagents were analytical reagent grade. The asphalt used was AH-90, which was provided by Dongming Petrochemical Company, Dongming, Shandong, China. The mineral aggregate was the mixture of different size particles of marble stone. The FTIR spectrum was measured at 400 to $4000 \mathrm{~cm}^{-1}$ wavenumber range with averaging 16 scans at a resolution of $4 \mathrm{~cm}^{-1}$ on a Tensor-27 FTIR spectrophotometer (Bruker, Germany). The ${ }^{1} \mathrm{H}$ NMR spectrum was measured on a Bruker Avance 300M NMR spectrometer (Bruker, Germany), using $\mathrm{CDCl}_{3}$ as solvent and TMS as internal reference. Chemical shifts $(\delta)$ were given in ppm. Surface tension of asphalt emulsifier solutions was measured with Krüss Processor Tensiometer K12 (Krüss GmbH, Hamburg, Germany). Elemental analysis was measured by the Elementar vario EL type III element analyzer (Elementar, German). 


\subsection{Synthesis of asphalt emulsifier}

Scheme 1 shows the synthesis step of the cationic asphalt emulsifier.

$\mathrm{C}_{11} \mathrm{H}_{23} \mathrm{CONH}\left(\mathrm{CH}_{2}\right)_{3} \mathrm{~N}\left(\mathrm{CH}_{3}\right)_{2}+\underset{\mathrm{O}}{\mathrm{CH}_{2} \mathrm{CHCH}_{2} \mathrm{Cl} \longrightarrow}$

$\mathrm{C}_{11} \mathrm{H}_{23} \mathrm{CONH}\left(\mathrm{CH}_{2}\right)_{3} \stackrel{+}{\mathrm{N}}\left(\mathrm{CH}_{3}\right)_{2} \mathrm{CH}_{2} \mathrm{CH}-\underset{O}{/} \mathrm{CH}_{2} \cdot \mathrm{Cl}^{-}$

Intermediate (a)

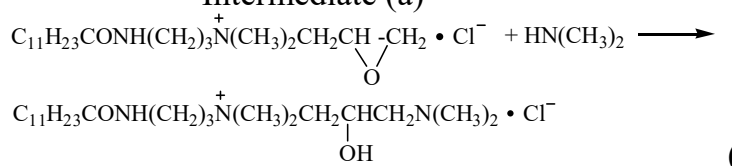

Product (b)

Scheme 1. Synthetic step of the asphalt emulsifier.

$\mathrm{N}, \mathrm{N}$-dimethyl-N-(ethylene oxide-2-methylene)coconut oil amide propyl-1-ammonium chloride (a): Coconut oil acyl propyl dimethyl tertiary amine (14.225 g, $0.05 \mathrm{~mol}$ ) was dissolved in $16 \mathrm{~mL}$ anhydrous ethanol. Then, epoxy chloropropane $(4.856 \mathrm{~g}, 0.0525 \mathrm{~mol})$ was added in it. The mixture was stirred for $5 \mathrm{~h}$ at $50^{\circ} \mathrm{C}$ in a water bath. The solvent in the mixed solution was removed by vacuum distillation. The intermediate (a) was recrystallized by anhydrous diethyl ether for three times, and dried in vacuum for 12 hours.

$\mathrm{N}, \mathrm{N}$-dimethyl-N-(3-(N',N'-dimethyl

amido)-2hydroxy propyl)-coconut oil amide propyl-1-ammonium chloride (b): $33 \%$ dimethylamine aqueous solution (17.1 $\mathrm{g}, 0.125 \mathrm{~mol}$ ) was added in intermediate (a) synthesized above. The mixture was stirred for $3 \mathrm{~h}$ at $65^{\circ} \mathrm{C}$ in a water bath. The solvent in the mixed solution was removed by vacuum distillation. The product (b) was recrystallized by acetone for three times, and dried in vacuum for 12 hours.

\subsection{The yield of intermediate (a)}

The yield $(Y)$ of intermediate (a) was obtained by Mohr analysis method. For reaction (R1), chloride ion was generated. $1.5000 \mathrm{~g}$ sample was weighed into a conical flask, then $50 \mathrm{~mL}$ distilled water and $0.1 \mathrm{~mL}$ of $5 \%$ potassium chromate indicator was added. The solution was titrated with $0.1 \mathrm{~mol} / \mathrm{L} \mathrm{AgNO}_{3}$ standard solution. The titration endpoint is the appearance of red-brown silver chromate. A blank titration was carried out at the same time.

$Y=\frac{N_{1} \times\left(V_{1}-V_{01}\right) \times m}{1000 \times m_{1} \times n_{1}} \times 100 \%$

where, $V_{1}$ means the consumed volume of silver nitrate standard solution, $\mathrm{mL}$. $V_{01}$ means the consumed volume of silver nitrate standard solution in blank titration, $\mathrm{mL}$. $m$ means the total mass of sample, gram. $m_{1}$ means the mass of sample to be titrated, gram. $N_{1}$ means the concentration of silver nitrate standard solution, $\mathrm{mol} / \mathrm{L} . n_{1}$ means the mole of epoxy chloropropane, mol.

\subsection{Epoxy group value}

The epoxy group value $(E)$ of intermediate (a) in reaction (R1) was measured according to the following method. $1.5000 \mathrm{~g}$ sample was weighed into a conical flask, then
$10 \mathrm{~mL}$ concentrated hydrochloric acid ( $\mathrm{HCl}$ )-acetone solution (volume ratio $1: 40$ ) and $10 \mathrm{~mL}$ ethanol were added. The solution was reflux at $70{ }^{\circ} \mathrm{C}$ for $1 \mathrm{~h}$, and then was cooled to room temperature. $0.1 \mathrm{~mL}$ of phenolphthalein indicator was added. The solution was titrated with standard sodium hydroxide solution $(0.1$ $\mathrm{mol} / \mathrm{L}$ ). The titration endpoint was the appearance of pink color. A blank determination was carried out at the same time.

$$
E=\frac{N_{2} \times\left(V_{2}-V_{02}\right) \times m}{1000 \times m_{2} \times n_{2}} \times 100 \%
$$

Where, $V_{2}$ means the consumed volume of sodium hydroxide standard solution, mL. $V_{02}$ means the consumed volume of sodium hydroxide standard solution in blank determination, mL. $m$ means the total mass of sample, gram. $m_{2}$ means the mass of the sample to be titrated, gram. $N_{2}$ means the concentration of sodium hydroxide standard solution, mol/L. $n_{2}$ means the mole of PKO, mol.

\subsection{Surface tension and $\mathrm{CMC}$}

Surface tension of the asphalt emulsifier solutions decreases sharply with the increase of concentration, and nearly does not change when the concentration gets CMC. The critical micelle concentration and the surface tension at $\mathrm{CMC}$ were at the turning point of the curve of surface tension to logarithm of concentration.

\subsection{Performance test of bituminous emulsion}

\subsubsection{Preparation of bituminous emulsion}

$15.0 \mathrm{~g}$ asphalt emulsifier was dissolved in $200 \mathrm{~g}$ water, then the solution was heated to $65{ }^{\circ} \mathrm{C}$. The $\mathrm{pH}$ value of the asphalt emulsifier aqueous solution was adjusted to 2.0 by adding dilute $\mathrm{HCl}(1: 1) .300 \mathrm{~g}$ asphalt (AH-90) was heated to $125{ }^{\circ} \mathrm{C}$. The bituminous emulsion was obtained by mixing the asphalt emulsifier aqueous solution and the asphalt at a colloid mill for $1 \mathrm{~min}$.

\subsubsection{Mixing experiment}

$100 \mathrm{~g}$ Mineral aggregate, $1 \mathrm{~g}$ cement and $8 \mathrm{~g}$ water were mixed in a bowl. Then, $11 \mathrm{~g}$ bituminous emulsion was added, and the mixture was stirred at a speed of 60 revolutions per minute. The mixing time was measured by observation of the mixing appearance. The room temperature was $23.0{ }^{\circ} \mathrm{C}$.

\section{Results and discussion}

\subsection{Synthesis process optimization}

\subsubsection{Feedstock mole ratio}

The reaction conditions were fixed at: reaction temperature $50{ }^{\circ} \mathrm{C}$, reaction time $5 \mathrm{~h}$. Figure 1 gives the 
yield $(Y)$ of intermediate (a) versus feedstock mole ratio of epichlorohydrin to coconut oil acyl propyl dimethyl tertiary amine $(m)$. As shown in Fig. 1, the yield increases with the feedstock mole ratio $(m)$. Then, the yield decreases as the feedstock mole ratio greater than 1.05. Figure 2 represents the epoxy group value $(E)$ of intermediate (a) versus feedstock mole ratio of epichlorohydrin to coconut oil acyl propyl dimethyl tertiary amine $(m)$. As shown in Fig. 2, the epoxy group value increases with the feedstock mole ratio $(m)$. So, the optimum feedstock mole ratio $(m)$ is selected as 1.05 .

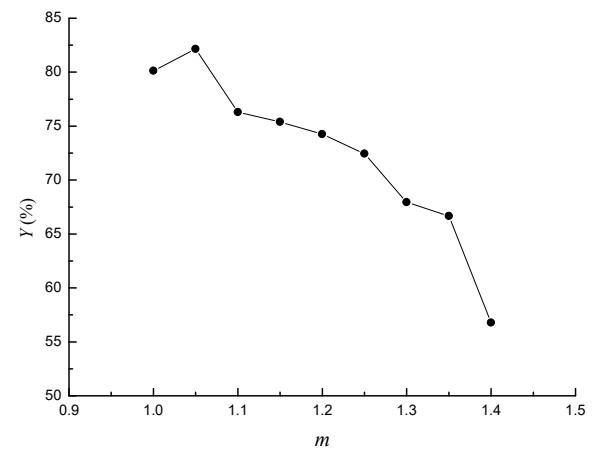

Figure 1. Yield vs. feedstock mole ratio.

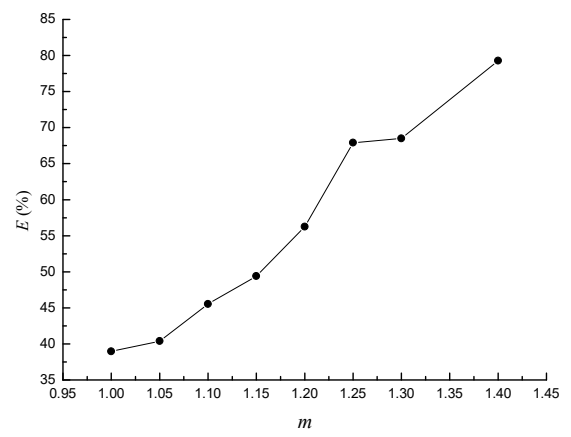

Figure 2. Epoxy group value vs. feedstock mole ratio.

\subsubsection{Reaction time}

The reaction conditions were fixed at: reaction temperature $50{ }^{\circ} \mathrm{C}$, feedstock mole ratio of epichlorohydrin to coconut oil acyl propyl dimethyl tertiary amine 1.05 . Figure 3 gives the yield $(Y)$ versus reaction time $(t)$. As shown in Fig. 3, the yield increases with the reaction time and gets maximum value at $6 \mathrm{~h}$, then decreases with the reaction time. Figure 4 represents the epoxy group value $(E)$ of intermediate (a) versus reaction time. As shown in Fig. 4, the epoxy group value increases with reaction time in the first stage, then decreases as the reaction time greater than $4 \mathrm{~h}$. The decreasing of epoxy group value is of the reason of the hydrolysis and the ring opening of the epoxy group. So, the optimum reaction time is selected as $5 \mathrm{~h}$.

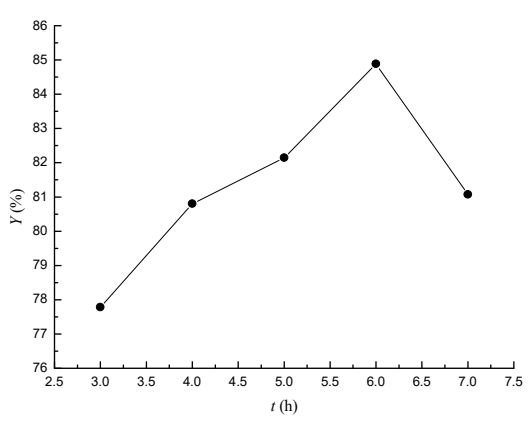

Figure 3. Yield vs. reaction time.

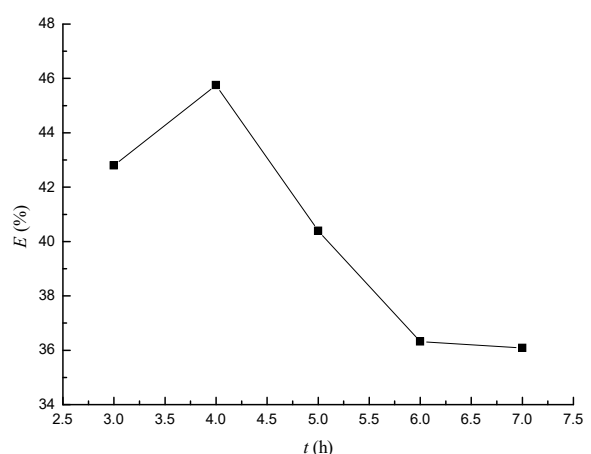

Figure 4. Epoxy group value vs. reaction time.

\subsubsection{Reaction temperature}

The reaction conditions were fixed at: reaction time $5 \mathrm{~h}$, feedstock mole ratio of epichlorohydrin to coconut oil acyl propyl dimethyl tertiary amine 1.05 . Figure 5 gives the yield $(Y)$ versus reaction temperature $(T)$. As shown in Fig. 5, the yield increases with the reaction temperature and gets maximum value at $60{ }^{\circ} \mathrm{C}$, then decreases with the reaction temperature. Figure 6 represents the epoxy group value $(E)$ of intermediate (a) versus reaction temperature. As shown in Fig. 6, the epoxy group value decreases with the reaction temperature. The decreasing of epoxy group value is of the reason of the hydrolysis and the ring opening of the epoxy group at higher reaction temperature. So, the optimum reaction temperature is selected as $50{ }^{\circ} \mathrm{C}$.

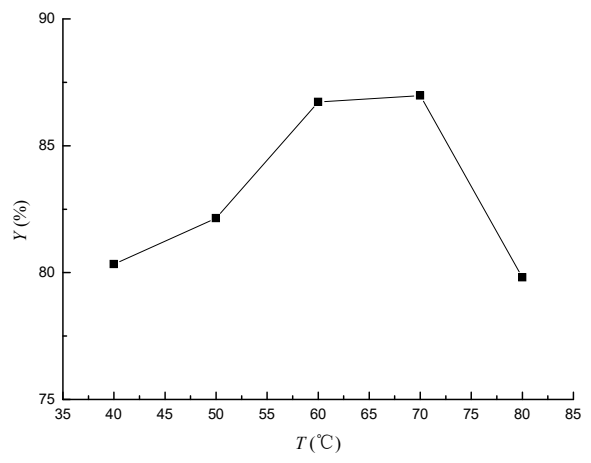

Figure 5. Yield vs. reaction temperature. 


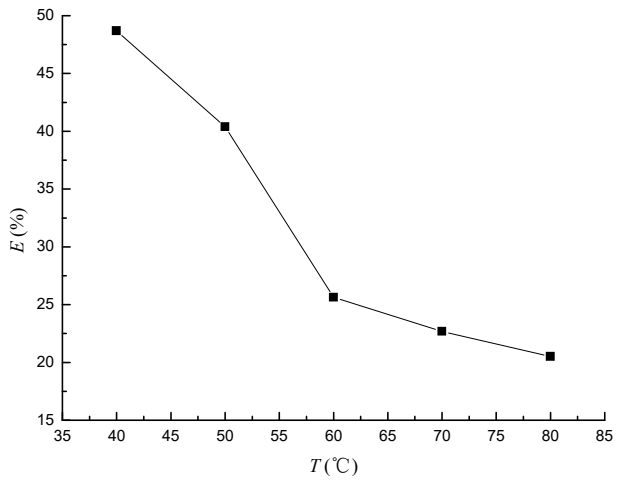

Figure 6. Epoxy group value vs. reaction temperature.

Therefore, the optimum reaction conditions can be got: reaction temperature $50{ }^{\circ} \mathrm{C}$, feedstock mole ratio of epichlorohydrin to coconut oil acyl propyl dimethyl tertiary amine 1.05 , reaction time $5 \mathrm{~h}$. The yield of intermediate (a) is $82.15 \%$, the epoxy group value is $40.39 \%$ at the optimum reaction conditions.

\subsection{FTIR characterization}

Figure 7 is the FTIR spectrum of intermediate (a). The absorption at $3292 \mathrm{~cm}^{-1}$ (peak 1) originates from the N-H stretching in amide. The absorption at $3015 \mathrm{~cm}^{-1}$ (peak 2) originates from the $\mathrm{C}-\mathrm{H}$ stretching in epoxy group. The absorption at $2800-3100 \mathrm{~cm}^{-1}$ originates from the $\mathrm{C}-\mathrm{H}$ stretching. The absorption at $2920 \mathrm{~cm}^{-1}$ (peak 3) originates from the methyl asymmetrical stretching. The absorption at $2853 \mathrm{~cm}^{-1}$ (peak 4) originates from the methyl symmetrical stretching. The absorption at 1645 $\mathrm{cm}^{-1}$ (peak 5) originates from the $\mathrm{C}=\mathrm{O}$ stretching in amide. The absorption at $1549 \mathrm{~cm}^{-1}$ (peak 6) originates from the $\mathrm{N}-\mathrm{H}$ bending in amide. The absorption at 1466 $\mathrm{cm}^{-1}$ (peak 7) originates from the $\mathrm{C}-\mathrm{N}$ stretching in amide. The absorption at $1260 \mathrm{~cm}^{-1}$ (peak 8) originates from the $\mathrm{C}-\mathrm{O}$ stretching in epoxy group. The absorption at $964 \mathrm{~cm}^{-}$ ${ }^{1}$ (peak 9) originates from the asymmetrical stretching of epoxy group. The absorption at $720 \mathrm{~cm}^{-1}$ (peak 10) originates from the $\mathrm{CH}_{2}$ in-plane swing vibration. The absorption at $600 \mathrm{~cm}^{-1}$ (peak 11) originates from the N-H out-of-plane bending.

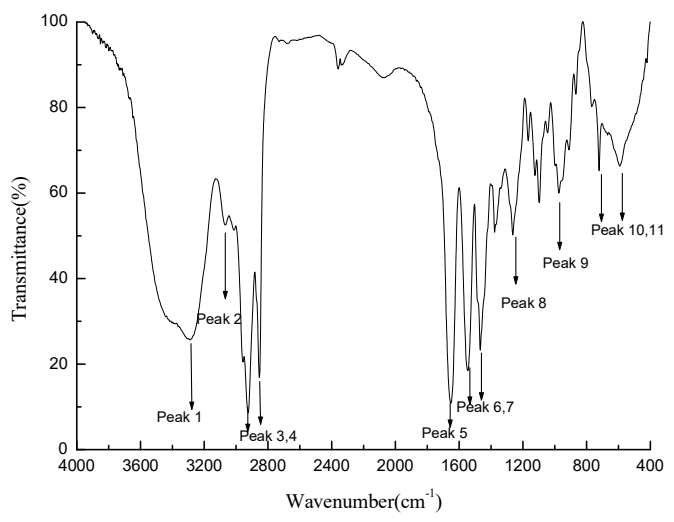

Figure 7. The FTIR spectrum of intermediate (a).
Figure 8 is the FTIR spectrum of asphalt emulsifier (b). The absorption at $3600-3216 \mathrm{~cm}^{-1}$ (peak 1) originates from the $\mathrm{O}-\mathrm{H}$ stretching and $\mathrm{N}-\mathrm{H}$ asymmetrical stretching. The absorption at $3078 \mathrm{~cm}^{-1}$ (peak 2) originates from the $\mathrm{N}-\mathrm{H}$ stretching in amide. The absorption at $2928 \mathrm{~cm}^{-1}$ (peak 3) originates from the methyl asymmetrical stretching. The absorption at $2852 \mathrm{~cm}^{-1}$ (peak 4) originates from the methyl symmetrical stretching. The absorption at $1640 \mathrm{~cm}^{-1}$ (peak 5) originates from the $\mathrm{C}=\mathrm{O}$ stretching in amide. The absorption at $1546 \mathrm{~cm}^{-1}$ (peak 6) originates from the N-H bending in amide. The absorption at $1460 \mathrm{~cm}^{-1}$ (peak 7) originates from the $\mathrm{C}-\mathrm{N}$ stretching in amide. The absorption at $1362 \mathrm{~cm}^{-1}$ (peak 8) originates from the methylene symmetrical bending. The absorption at $1250 \mathrm{~cm}^{-1}$ (peak 9) originates from the $\mathrm{N}-\mathrm{H}$ bending. The absorption at $1098 \mathrm{~cm}^{-1}$ (peak 10) originates from the $\mathrm{C}-\mathrm{O}$ stretching. The absorption at $720 \mathrm{~cm}^{-1}$ (peak 11) originates from the $\mathrm{CH}_{2}$ in-plane swing vibration. The absorption at $600 \mathrm{~cm}^{-1}$ (peak 12) originates from the N-H out-of-plane bending.

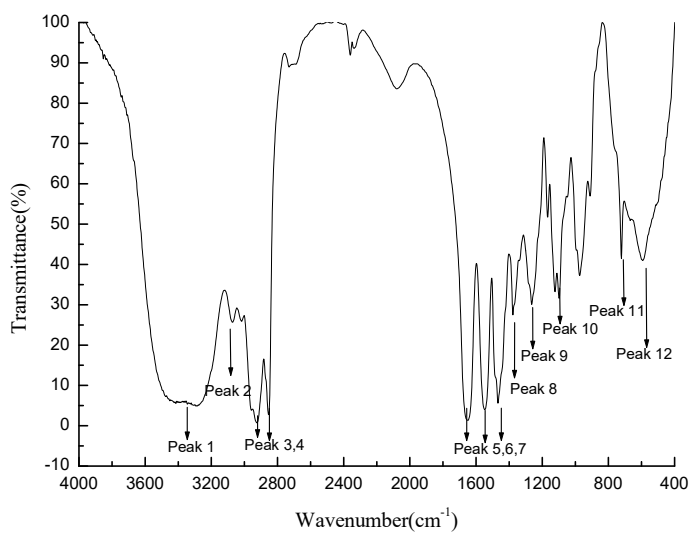

Figure 8. The FTIR spectrum of asphalt emulsifier (b).

\section{3 ${ }^{1} \mathrm{H}$ NMR analysis}

${ }^{1} \mathrm{H} \quad \mathrm{NMR}\left(\mathrm{CDCl}_{3}, \quad 300 \mathrm{~Hz}\right) \delta: 0.91\left(-\mathrm{C}_{3}, \quad \mathrm{t}, 3 \mathrm{H}\right) ; \quad 1.32(-$ $\left.\left(\mathrm{CH}_{2}\right)_{8^{-}}, \quad \mathrm{m}, 16 \mathrm{H}\right) ; \quad 1.57\left(\mathrm{C}_{9} \mathrm{H}_{19^{-}}-\mathrm{CH}_{2-}^{-}, \mathrm{s}, 2 \mathrm{H}\right) ; 2.05-2.20(-$ $\left.\mathrm{CONH}-\mathrm{CH}_{2}-\mathrm{CH}_{2-}, \mathrm{d}, 2 \mathrm{H}\right) ; 2.31\left(\mathrm{C}_{10} \mathrm{H}_{21}-\mathrm{CH}_{2-}, \mathrm{t}, 2 \mathrm{H}\right) ; 3.15-$ $3.70\left(-\mathrm{CH}_{2}-\mathrm{CH}_{2}-\mathrm{CH}_{2}-\mathrm{N}^{+}-\mathrm{CH}_{2}-\mathrm{CH}-\mathrm{CH}_{2}-, \quad \mathrm{m}, 9 \mathrm{H}\right) ; 3.71-$ $3.92\left(-\mathrm{N}^{+}-\left(\mathrm{CH}_{3}\right)_{2}, \mathrm{~m}, 6 \mathrm{H}\right)$; 7.90-8.09(-NH-, m,1H) ppm. Therefore, the results of ${ }^{1} \mathrm{H}$ NMR analysis conform to the chemical structure of the intermediate (a).

\subsection{Elemental analysis}

Anal.: Calcd. for intermediate (a): C, 63.75\%; H, 10.89\%; N, 7.44\%. Found: C, $57.00 \%$; H, $11.63 \%$; N, $6.94 \%$.

\subsection{CMC and surface tension}

Figure 9 gives the surface tension $(\sigma)$ versus the logarithm of the asphalt emulsifier concentration $(c)$ at $298 \mathrm{~K}$. The surface tension showed a shape decrease firstly, then kept at a constant level. The CMC of the asphalt emulsifier is $7.80 \times 10^{-2} \mathrm{~mol} / \mathrm{L}$. The surface tension at $\mathrm{CMC}$ is $24.57 \mathrm{mN} / \mathrm{m}$. 


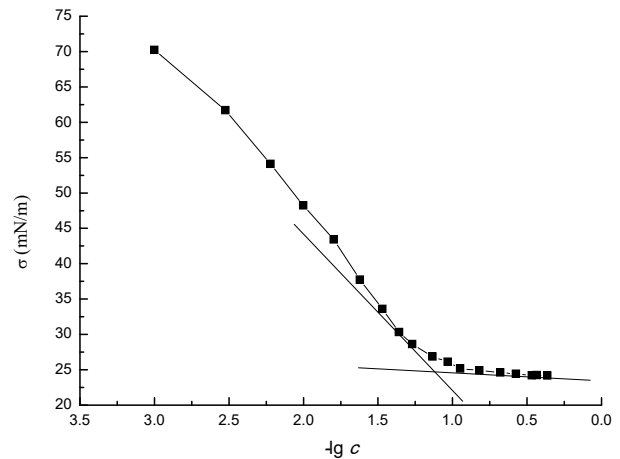

Figure 9. Surface tension vs. the logarithm of concentration.

\subsection{Performance test of bituminous emulsion}

The cationic asphalt emulsifier has good emulsifying ability for AH-90 asphalt. The bituminous emulsion to be prepared had higher storage stability. There was no separation of aqueous phase and asphalt phase appeared after 5 days. The mixing time was $60 \mathrm{~s}$, which indicated that the emulsifier is a medium-set asphalt emulsifier. It can be applied to the application of tack coat, fog seal and chip seal in asphalt pavement construction.

\section{Summary}

A new cationic asphalt emulsifier was synthesized by coconut oil acyl propyl dimethyl tertiary amine (PKO), epoxy chloropropane and dimethylamine. The optimum reaction condition, the critical micelle concentration (CMC) and the surface tension at CMC of the asphalt emulsifier were obtained. The emulsifier belongs to a medium-set asphalt emulsifier. It can be applied to the application of tack coat, fog seal and chip seal in asphalt pavement construction.

\section{Acknowledgement}

The authors would like to thank the financial support from the Shandong Provincial Science and Technology Project (No. 2015GGX107005).

\section{References}

1. R.A. Mercado, J.L. Salager, V. Sadtler, et al. Breaking of a cationic amine oil-in-water emulsion by $\mathrm{pH}$ increasing: Rheological monitoring to modelize asphalt emulsion rupture. Colloids and Surfaces A: Physicochemical and Engineering Aspects, 458:63-68(2014)

2. F. Yang, G. Li, J. Qi, et al. Synthesis and surface activity properties of alkylphenol polyoxyethylene nonionic trimeric surfactants. Applied Surface Science, 257(1): 312-318(2010)

3. K. Isobe, R. Tamaki. Nonionic emulsifier for asphalt. U.S. Patent 6,114,418. (2000)
4. P.R.J. Blanpain, J.W. Creak, G.F. Mori, et al. Cationic asphalt emulsions. U.S. Patent 4,997,868. (1991)

5. W.A. Mclntosh. Alkali lignin to stabilize slow-break asphalt emulsions. Industrial \& Engineering Chemistry, 44(7): 1656-1659(1952)

6. N.A. Negm, M.A. El-Hashash, D.E. Mohamed, et al. Gemini cationic surfactants: synthesis and influence of chemical structure on the surface activity. Journal of Surfactants and Detergents, 16(5): 733-738(2013)

7. L. Shi, Y. Zhao. Synthesis and performance evaluation of a Gemini cationic asphalt emulsifier. Petroleum asphalt, 24(6): 66-71(2010)

8. H.P. Li, H. Zhao, K. Liao. The preparation of asphalt emulsions with dissymmetric Gemini quaternary ammonium salts cationic surfactants. Energy Sources, Part A: Recovery, Utilization, and Environmental Effects, 35(24): 2285-2293(2013)

9. T. Banno, K. Toshima, K. Kawada, et al. Synthesis and properties of gemini-type cationic surfactants containing carbonate linkages in the linker moiety directed toward green and sustainable chemistry. Journal of Surfactants and Detergents, 12(3): 249259(2009)

10. L. Shi, M. Sun, N. Li, B. Zhang. A novel betaine type asphalt emulsifier synthesized and investigated by online FTIR spectrophotometric method. Chem. Ind. Chem. Eng. Q., 21, 113-121(2015)

11. X. Tao, L. Shi, M. Sun, N. Li. Synthesis of lignin amine asphalt emulsifier and its investigation by online FTIR spectrophotometry. Advanced Materials Research, 909, 72-76(2014)

12. N. Li, L. Shi, X. Gong, Q. Xu, X. Liu, X. Wang. Synthesis of a novel cationic asphalt emulsifier and its investigation by online FTIR spectrophotometry. Res. Chem. Intermed., 41(4), 1935-1950(2015)

13. C. Huai, L. Shi, N. Li. Synthesis of a novel betainetype asphalt emulsifier and its investigation by online FTIR spectrophotometry. Research on Chemical Intermediates, 39, 597-614(2013)

14. Xiao-Meng Yu, Lai-Shun Shi, Cun-Fei Ma, Lu Hou, Jing-Wen Kong, Ya-Li Sun. The synthesis and characterization of novel bis-cationic asphalt emulsifier. MATEC Web of Conferences, 130: 04004, 80-84(2017) 